\title{
Radio-cephalic fistula recovered with drainage to forearm basilic vein
}

\author{
António NORTON DE MATOS, ${ }^{1,2}$ Clemente Neves SOUSA, , ,3,4 Paulo ALMEIDA, , ${ }^{1,2}$ \\ Paulo TELES, ${ }^{5}$ Luís LOUREIRO, ${ }^{1,2}$ Gabriela TEIXEIRA, ${ }^{1,2}$ Duarte REGO, ${ }^{1,2}$ \\ Sérgio TEIXEIRA ${ }^{1,2}$ \\ ${ }^{1}$ Vascular Access Center (Grupo Estudos Vasculares-GEV), Porto, Portugal; ${ }^{2}$ Angiology and Vascular \\ Surgery Departament, Centro Hospitalar do Porto, Portugal; ${ }^{3}$ Nursing School of Porto, Porto, Portugal; \\ ${ }^{4}$ CINTESIS - Center for Health Technology and Services Research Faculty of Medicine, Porto University; \\ ${ }^{5}$ School of Economics and LIAAD-INESC Porto LA, Porto University, Porto, Portugal
}

\begin{abstract}
Dysfunction problems with vascular access are a concern to patients and dialysis units. The vascular surgeon should analyse such dysfunction and perform a careful assessment of the vascular network in order to find new fistula layouts. We introduce and discuss the case of creation of a radiocephalic fistula with outflow into the forearm basilic vein through rotation of the forearm basilic vein toward the cephalic vein in the forearm of an 88-year-old hemodialysis male patient. This technique enables extending fistula patency and improves cost efficiency.
\end{abstract}

Key words: Vascular access, hemodialysis, arteriovenous fistula, forearm basilic vein

\section{INTRODUCTION}

The radio-cephalic arteriovenous fistula (RC-AVF) is the first choice for vascular access in patients requiring hemodialysis. ${ }^{1,2}$ This type of vascular access has fewer complications (steal syndrome, high flow, or venous hypertension syndrome) when compared with fistulas in other sites.

RC-AVF patency is usually high when clinical/physical examination and preoperative and postoperative ultrasound are used carefully. Monitoring and surveillance programs in dialysis units have shown improvement in access patency and a decrease in hospital admissions caused by access dysfunction. ${ }^{3}$ However, some RC-AVF may cause dysfunction problems difficult to solve, raising great challenges to vascular surgeons. Most common

Correspondence to: A. N. de Matos, MD, Grupo Estudos Vasculares, Rua Ceuta 118,3 S/22, 4050-190 Porto, Portugal. E-mail: a.nortondematos@gmail.com Conflict of Interest: The authors do not have any conflict of interest and were not funded.

Disclosure of grants or other funding: None. solutions cannot be performed in some RC-AVF, because of fistula limited arterial outflow, decrease or absence of venous network, problems in arterial network or because of all these reasons. ${ }^{4}$ The forearm basilic vein is a good solution for correction of AVF problems.

Patients with complex AVF require personal approaches and specific solutions. In this report, a surgical approach to salvage an RC-AVF with outflow occlusion using the forearm basilic vein is introduced.

\section{CASE REPORT}

We report the case of an 88-year-old male patient with hereditary nephropathy as kidney disease etiology and without comorbidities. A RC-AVF with side-to-end anastomosis was created on his left arm in April 2010 and he began hemodialysis treatment through this fistula in November 2010.

The patient was referred to our vascular access center in July 2015, because the access had a 30\%-40\% recirculation rate, high venous pressure, and problems in the venous cannulation. 


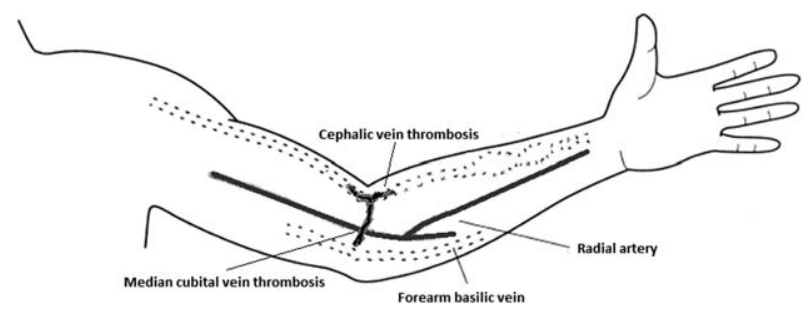

Figure 1 Radio-cephalic fistula with thrombosis in the cephalic vein and the median cubital vein in the elbow.

Physical examination detected that the fistula had two aneurysms in the cannulation sites. The forearm had collateral circulation after the venous cannulation aneurysm. The fistula had no thrill in the draining vein and only had hyperpulsatile pulse. The cephalic vein and the median cubital vein were hard to compress in the elbow.

Ultrasound evaluation showed that the size of the anastomotic connection was $5 \mathrm{~mm}$ and that the brachial artery flow was $400 \mathrm{~mL} / \mathrm{minutes}$. When we started, the arm cephalic vein and the median cubital vein were occluded/thrombosed in the elbow (Figure 1). The fistula had no outflow flux into the arm, draining was made into the forearm collateral veins. The forearm basilic and the basilic veins diameter was 4 and $5 \mathrm{~mm}$, respectively.

The fistula condition was not appropriate for a thrombectomy by angioplasty to be carried out because the occlusion process or thrombosis was not recent. Therefore, we decided to isolate the forearm basilic vein and rotate it toward the cephalic vein (Figure 2). The fistula outflow passes through the cephalic vein into the forearm basilic vein which in turn drains into the arm basilic vein (Figure 3). The fistula was cannulated with success in the first postoperative day.

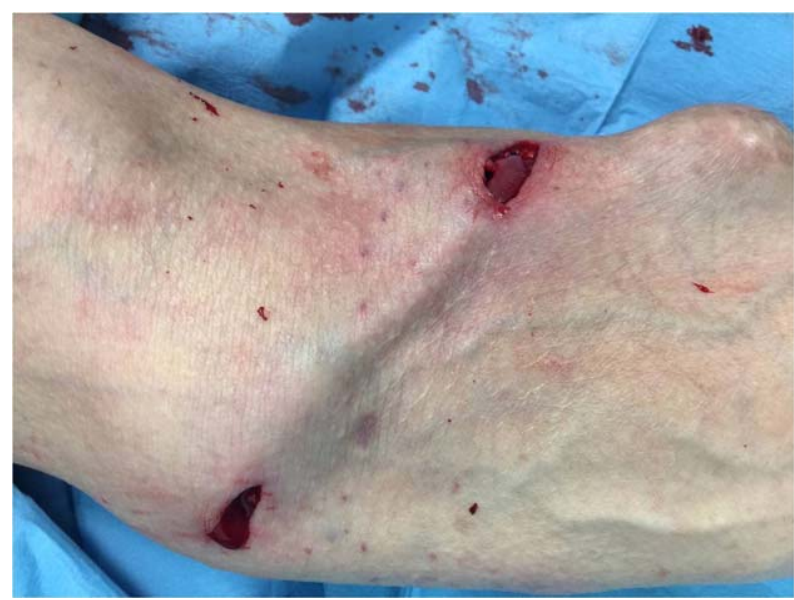

Figure 3 Radio-cephalic fistula recovered with drainage into forearm basilic vein.

The fistula is currently working free of problems or complications and the patient preserves the possibility of basilic vein transposition on the left arm.

\section{DISCUSSION}

The patient started hemodialysis by RC-AVF and, after five years of treatment, he was referred to our vascular access center because the access had problems in the venous cannulation. The cephalic vein and the median cubital vein were thrombosed in the elbow with no recovery possible, which led to the development of the venous network in the forearm. The patient had a forearm basilic vein with $4 \mathrm{~mm}$ diameter which was dissected and rotated towards the forearm cephalic vein.

We could not find any description of such type of arteriovenous access in the literature. However, Sousa et al. ${ }^{4}$ used in 2016 the forearm basilic vein as outflow of the
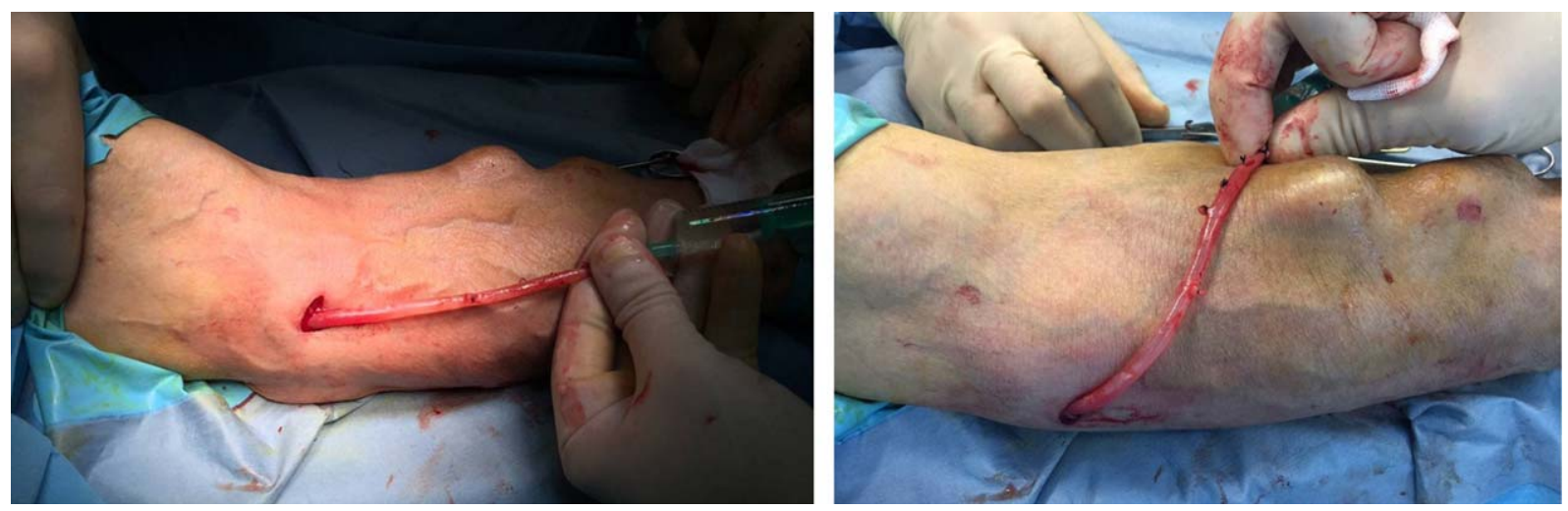

Figure 2 Radio-cephalic fistula with rotation of the forearm basilic vein towards the cephalic vein. 
brachial-cephalic fistula. In their procedure, the forearm cephalic vein was rotated towards the forearm basilic vein. We decided to analyze the vascular network of the left arm with ultrasound, in order to identify the best solution for arteriovenous access. The forearm basilic vein was dissected and rotated toward the forearm cephalic vein. The dissection of the forearm basilic vein must be made carefully in order to avoid possible vessel injury and venous spasm. ${ }^{5}$ Using the forearm basilic vein as outflow of the RC-AVF enabled access patency maintenance. The solution found allows extending the use of the fistula without requiring a central venous catheter or another fistula, preserving the vascular network for future accesses, arterialization of the arm basilic vein and contributing to increase its diameter. ${ }^{4}$ The basilic vein has currently a $5 \mathrm{~mm}$ diameter which can ease its future transposition in a single stage.

The use of the forearm basilic vein or the creation of an ulnar-basilic arteriovenous fistula (UB-AVF) is not popular and remains a rarely performed fistula. American or European guidelines about vascular access do not mention the UB-AVF $^{2,5}$ and a thorough review work identified only 8 studies until 2014 with a total of 274 UB-AVFs. ${ }^{6}$ The experience with UB-AVFs is still short, but vascular surgeons have to detect and identify patients possessing the appropriate conditions for this type of fistula or segments of vein that can be used in fistula reconstruction.

When a complex access has a dysfunction, we recommend that the information concerning the technique used to solve it is sent to the dialysis unit together with photos of the surgery, if possible. This approach will enable the dialysis nurse to understand how access is working and to identify the appropriate cannulation sites.

In our case, we identified the site where the venovenous anastomosis (forearm basilic vein with cephalic vein) was carried out and flagged it to prevent it from being cannulated again. Educating the dialysis nurse to always perform cannulation in the forearm and never in the basilic vein is crucial.

Currently, the AVF is 18 months old with no dysfunction or cannulation problems. The drainage vein kept a length of more than $14 \mathrm{~cm}$, perfect for application of the rope-ladder cannulation technique. ${ }^{7}$ However, a vascular access surveillance program should be implemented by the dialysis unit $^{8}$ whose staff should include dialysis nurses with training to identify access problems ${ }^{9,10}$ and to select the appropriate cannulation technique. We think this procedure is appropriate when the forearm basilic vein can be rotated towards the forearm cephalic vein.

\section{CONCLUSION}

This type of arteriovenous fistula can be a good option when the forearm basilic vein has an appropriate diameter and allows the rotation toward the cephalic vein. Creation of this type of fistula does not raise any technical difficulties but a correct dissection of the forearm basilic vein is required. This procedure enables recovering the fistula without use of the arm veins and the simultaneous arterialization of the basilic vein.

Manuscript received October 2016; revised November 2016.

\section{REFERENCES}

1 Kukita K, Ohira S, Amano I, et al. 2011 update Japanese society for dialysis therapy guidelines of vascular access construction and repair for chronic hemodialysis. Ther Apher Dial. 2015; 19(Suppl 1): 1-39.

2 NKF-K/DOQI. Foundation national kidney. K/DOQI clinical practice guidelines in vascular access: 2006 update. Am J Kidney Dis. 2006; 48(Suppl): S176-S306.

3 Tessitore N, Bedogna V, Lipari G, et al. Bedside screening for fistula stenosis should be tailored to the site of the arteriovenous anastomosis. Clin J Am Soc Nephrol. 2011; 6: 1073-1080.

4 Sousa CN, Norton de Matos A, Rocha A, Almeida P, Xavier E. Brachial-cephalic fistula recovered with drainage for the basilic vein: Case report. Hemodial Int. 2016; 20: E15-E17.

5 Cavatorta F, Galli S, Zollo A, Crespi E, Carnabuci A. Ulno-basilic arteriovenous fistulae: Indications and surgical technique. J Vasc Access. 2008; 9: 73-80.

6 Tordoir J, Canaud B, Haage P, et al. EBPG on vascular access. Nephrol Dial Transplant. 2007; 22(Suppl 2): ii88-iil17.

7 Al Shakarchi J, Khawaja A, Cassidy D, Houston JG, Inston N. Efficacy of the Ulnar-Basilic arteriovenous fistula for hemodialysis: A systematic review. Ann Vasc Surg. 2016; 32:1-4.

8 van Loon MM, Kessels AG, Van der Sande FM, Tordoir JH. Cannulation and vascular access-related complications in hemodialysis: Factors determining successful cannulation. Hemodial Int. 2009; 13: 498-504.

9 Sousa CN, Apóstolo JL, Figueiredo MH, Martins MM, Dias VF. Physical examination: How to examine the arm with arteriovenous fistula. Hemodial Int. 2013; 17: 300-306.

10 Sousa CN, Teles P, Dias VF, Apóstolo JL, Figueiredo MH, Martins MM. Physical examination of arteriovenous fistula: The influence of professional experience in the detection of complications. Hemodial Int. 2014; 18: 695-699. 